\title{
A STRONG LAW FOR THE SIZE OF YULE $m$-ORIENTED RECURSIVE TREES
}

\author{
MEHRI JAVANIAN AND MOHAMMAD Q. VAHIDI-ASL
}

\begin{abstract}
Let $N_{t}$ be the total number of nodes in a Yule $m$-oriented recursive tree at time $t$. Then $\left\{N_{t}: t \in\right.$ $[0, \infty)\}$ is a Yule process with birth rates $\lambda_{n}=(m(n-1)+1) \lambda$ for $n \geq 1$, where $N_{0}=1$. In this paper, we first give the exact distribution of $N_{t}$, then prove that

$$
\lim _{t \longrightarrow \infty} \frac{\ln N_{t}}{m \lambda t}=1, \quad \text { almost surely. }
$$
\end{abstract}

Mathematics Subject Classification 2000: 05C05

Additional Key Words and Phrases: Yule processes, recursive trees

\section{INTRODUCTION}

By a recursive tree we mean a labeled rooted tree such that each path from the root to any node of the tree is labeled with an increasing sequence of labels. Figure 1 shows all the recursive trees of size 4 .

A survey of applications and results on recursive trees is given in [6]. These trees are used, e.g., to model chain letters and pyramid schemes [5], and as a simplified growth model of the word wide web [1].

In [5], Recursive trees have been proposed as a model for chain letters, where a company is founded to spread a particular item (lottery tickets, good luck charm, etc.). The initial recruiter looks for a willing participant to buy a copy of the letter. The recruiter and the new letter holder compete with equal chance to recruit a new participant. The process proceeds in this way, where at each stage all existing participants compete with equal chance to attract the next participant in the scheme.

In working with recursive trees it is to convenient to consider an extension of these trees obtained by adding a different type of node called external at each possible insertion position.

Orientation in the plane was not taken into account in the definition of recursive trees. The two labeled trees in Figure 2 are only two drawings of the same recursive tree. If different orientations are taken to represent different trees, we arrive at a definition of a plane-oriented recursive tree. In such a tree, if a node has outdegree $d$; there are $d$ children under it, with $d+1$ external nodes.

Figure 3 shows the extension of the left tree in Figure 2, (the external nodes are

DOI 10.2478/v10294-012-0015-1

CUniversity of SS. Cyril and Methodius in Trnava 
M. JAVANIAN AND M. Q. VAHIDI-ASL
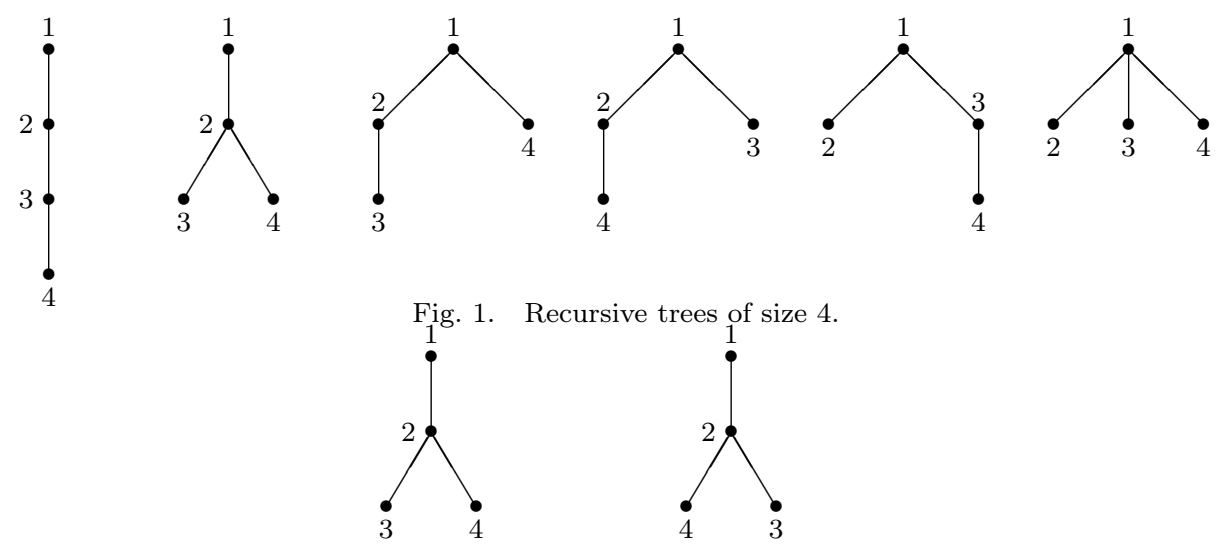

Fig. 2. Two different plane-oriented trees.

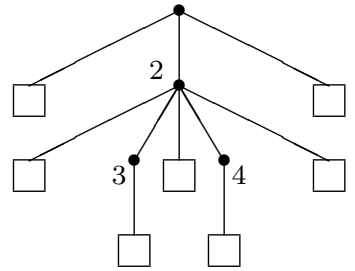

Fig. 3. An extended plane-oriented recursive tree.

shown as squares).

In this paper we consider $m$-oriented recursive trees the generalization of random plane-oriented recursive trees where if a node has outdegree $d$; there are $d$ children under it, with $(m-1) d+1$ external nodes, (this generalization first defined in [3]). If $d(i)$ denotes the outdegree of the $i$ th node then the total number of external nodes in an extended $m$-oriented recursive tree of size $n$ is

$$
\begin{aligned}
\sum_{i=1}^{n}((m-1) d(i)+1) & =(m-1) \sum_{i=1}^{n} d(i)+n \\
& =(m-1)(n-1)+n \\
& =m(n-1)+1 .
\end{aligned}
$$

A random $m$-oriented recursive tree of size $n$ is constructed as follows. One starts from a root node holding the label 1 ; at stage $i(i=2,3, \ldots, n)$ a new node holding label $i$ (the $i$ th node) is attached to any previous node $j$ of outdegree $d(j)$ of the already grown tree $T_{i-1}$ of size $i-1$ with probability $\frac{(m-1) d(j)+1}{m(i-2)+1}$ (the number of remaining external nodes for the node $j,(m-1) d(j)+1$, is divided by $m(i-2)+1$, the number of all external nodes). This function implies that the higher outdegree nodes possess a higher attraction for new neighbors.

We now consider the Yule recursive trees. We remind that a pure birth process $\left\{Y_{t}: t \in[0, \infty)\right\}$ with linear birth rates $\lambda_{n}=n \lambda$ for $n \geq 1$ is called a Yule process, (see Ross [7]).

Assume each external node in a random $m$-oriented recursive tree, independent 


\section{LAW FOR THE SIZE OF YULE $m$-ORIENTED RECURSIVE TREES}

of any other external nodes will be substituted by a new node, in a random time interval which has an exponential distribution with parameter $\lambda>0$. That is, when there are $n$ nodes in the tree, one of the $m(n-1)+1$ available external nodes will be substitute by a node in a random time $X_{i}, i=1, \ldots, m(n-1)+1$, where $X_{1}, \ldots, X_{m(n-1)+1}$ are i.i.d random variables with an exponential distribution of parameter $\lambda>0$. Suppose the tree has one node (the root) at time $T_{0}=0$, and $T_{n}$ is the time interval between the $(n-1)$ th and $n$th insertions. So the $(n+1)$ th node ( $n$th insertion) will be inserted in one of the $m(n-1)+1$ external nodes after the time interval $T_{n}=\min \left\{X_{1}, \ldots, X_{m(n-1)+1}\right\}$, which has an exponential distribution with parameter $(m(n-1)+1) \lambda$. Let $N_{t}$ be the number of nodes in the tree by time t. Then $\left\{N_{t}: t \in[0, \infty)\right\}$ is a Yule process with birth rates $\lambda_{n}=(m(n-1)+1) \lambda$ for $n \geq 1$, where $N_{0}=1$. The resulting random $m$-oriented recursive tree that grows in real time, with the nodes added as a Yule process with birth rates $\lambda_{n}=(m(n-1)+1) \lambda$ for $n \geq 1$, is a Yule m-oriented recursive tree.

In chain letter schemes the success of the recruits of a letter holder adds to his own profit. A letter holder gets a commission whenever anyone in his own subtree recruits. A measure of this success is the size of the subtree rooted at $i$ th node in a recursive tree. Also the size of a Yule tree will be a measure of the initial company's profit. So the size of Yule trees is an interesting parameter. Feng, and $\mathrm{Su}$ [4] have studied the size of Yule recursive trees. The size of Yule binary search trees is investigated by Chauvin, et. al, in [2]. Here we first give the exact distribution of the size of Yule $m$-oriented recursive tree. Then by using of the exact distribution of the size, we obtain an almost sure result for the size.

\section{THE EXACT DISTRIBUTION OF $N_{T}$}

To find the exact distribution of $N_{t}$, we shall use $f_{n}(t)$, the density function of $T_{1}+\cdots+T_{n}$ which is given in the following lemma.

LEMMA 1. For $t>0$,

$$
f_{n}(t)=\prod_{j=1}^{n-1} \frac{m j+1}{m j} \cdot \lambda e^{-\lambda t}\left(1-e^{-m \lambda t}\right)^{n-1} .
$$

Proof. Let $F_{n}(t)$ be the cumulative distribution function of $T_{1}+\cdots+T_{n}$. So by definition of $T_{i}$ 's, we have

$$
\begin{aligned}
F_{n}(t) & =\int_{0}^{t} \mathbf{P}\left(T_{1}+\cdots+T_{n} \leq t \mid T_{1}+\cdots+T_{n-1}=x\right) d F_{n-1}(x) \\
& =\int_{0}^{t} \mathbf{P}\left(T_{n} \leq t-x\right) d F_{n-1}(x) \\
& =\int_{0}^{t}\left(1-e^{-(m(n-1)+1) \lambda(t-x)}\right) d F_{n-1}(x) \\
& =F_{n-1}(t)-e^{-(m(n-1)+1) \lambda t} \int_{0}^{t} e^{(m(n-1)+1) \lambda x} d F_{n-1}(x)
\end{aligned}
$$

Taking derivatives of both sides of the last equality, we get a recurrence equation in $f_{n}(t)$, from which the result follows by induction. 
Theorem 1. For $n \geq 1$,

$$
\mathbf{P}\left(N_{t}=n\right)=\prod_{j=1}^{n-1} \frac{m j+1}{m j} \cdot \frac{e^{-\lambda t}\left(1-e^{-m \lambda t}\right)^{n-1}}{m(n-1)+1} .
$$

Proof. Since $\mathbf{P}\left(T_{1}+\cdots+T_{n} \leq t\right)=\mathbf{P}\left(N_{t} \geq n+1\right)$, then

$$
\begin{aligned}
\mathbf{P}\left(N_{t}=n\right) & =F_{n-1}(t)-F_{n}(t) \\
& =e^{-(m(n-1)+1) \lambda t} \int_{0}^{t} e^{(m(n-1)+1) \lambda x} f_{n-1}(x) d x .
\end{aligned}
$$

Now by substituting $f_{n-1}(x)$ from Lemma 1 , and calculating the easy integral, the theorem follows.

\section{MAIN RESULT}

In this section, we use the notation $g(t) \sim h(t)$ for asymptotic equivalence of the two functions $g(t)$ and $h(t)$, as $t \longrightarrow \infty$. Here we establish that $\ln N_{t} \sim m \lambda t$, as $t \longrightarrow \infty$ almost surely.

\section{THEOREM 2 .}

$$
\lim _{t \longrightarrow \infty} \frac{\ln N_{t}}{m \lambda t} \longrightarrow 1, \quad \text { almost surely. }
$$

Proof. Note that

$$
\left(1-e^{-m \lambda t}\right)^{e^{m \lambda t}} \longrightarrow \frac{1}{e},
$$

as $t \longrightarrow \infty$. Then for any $0<\varepsilon<1$,

$$
\begin{aligned}
& \mathbf{P}\left(\frac{\ln N_{t}}{m \lambda t}>1+\varepsilon\right) \\
& =\mathbf{P}\left(N_{t}>e^{m \lambda t(1+\varepsilon)}\right) \\
& <e^{-\lambda t} \sum_{n=\left\lfloor e^{m \lambda t(1+\varepsilon)}\right\rfloor}^{\infty} \prod_{j=1}^{n-1} \frac{m j+1}{m j} \cdot \frac{\left(1-e^{-m \lambda t}\right)^{n-1}}{m(n-1)+1} \\
& <\frac{e^{-\lambda t}}{\left\lfloor e^{m \lambda t(1+\varepsilon)\rfloor}\right.} \sum_{n=\left\lfloor e^{m \lambda t(1+\varepsilon)}\right\rfloor}^{\infty} \prod_{j=1}^{n-1} \frac{m j+1}{m j} \cdot\left(1-e^{-m \lambda t}\right)^{n-1} \\
& =\frac{e^{-\lambda t}}{\left\lfloor e^{m \lambda t(1+\varepsilon)}\right\rfloor} \prod_{j=1}^{\left\lfloor e^{m \lambda t \varepsilon}\right\rfloor} \frac{m j+1}{m j} \\
& \times \sum_{n=\left\lfloor e^{m \lambda t(1+\varepsilon)}\right\rfloor}^{\infty} \prod_{j=\left\lfloor e^{m \lambda t \varepsilon}\right\rfloor+1}^{n-1} \frac{m j+1}{m j} \cdot\left(1-e^{-m \lambda t}\right)^{n-1} \\
& \sim \frac{e^{-\lambda t}}{e^{m \lambda t(1+\varepsilon)}} \prod_{j=1}^{\left\lfloor e^{m \lambda t \varepsilon}\right\rfloor} \frac{m j+1}{m j} \sum_{n=\left\lfloor e^{m \lambda t(1+\varepsilon)}\right\rfloor}^{\infty}\left(1-e^{-m \lambda t}\right)^{n-1}
\end{aligned}
$$


LAW FOR THE SIZE OF YULE $m$-ORIENTED RECURSIVE TREES

$$
\begin{aligned}
& \sim e^{-\lambda t(1+m \varepsilon)}\left(1-e^{-m \lambda t}\right)^{e^{m \lambda t(1+\varepsilon)}} \prod_{j=1}^{\left\lfloor e^{m \lambda t \varepsilon} \frac{m j+1}{m j}\right.} \\
& =e^{-\lambda t(1+m \varepsilon)}\left(\left(1-e^{-m \lambda t}\right)^{e^{m \lambda t}}\right)^{e^{m \lambda t \varepsilon}} \prod_{j=1}^{\left\lfloor e^{m \lambda t \varepsilon}\right\rfloor} \frac{m j+1}{m j} \\
& \sim e^{-\lambda t(1+m \varepsilon)} \cdot \frac{1}{e^{e^{m \lambda t \varepsilon}}} \prod_{j=1}^{\left\lfloor e^{m \lambda t \varepsilon\rfloor} \frac{m j+1}{m j}\right.} \\
& <e^{-\lambda t(1+m \varepsilon)}, \quad\left(\because \prod_{j=1}^{\left\lfloor e^{m \lambda t \varepsilon}\right\rfloor} \frac{m j+1}{m j}<\left(\frac{m+1}{m}\right)^{\left\lfloor e^{m \lambda t \varepsilon}\right\rfloor}<e^{e^{m \lambda t \varepsilon}}\right) .
\end{aligned}
$$

Therefore, there exists a sufficiently large natural number $n_{0}$ such that

$$
\mathbf{P}\left(\frac{\ln N_{n}}{m \lambda n}>1+\varepsilon\right)<\frac{1}{e^{\lambda n(1+m \varepsilon)}},
$$

for all $n>n_{0}$. Hence,

$$
\sum_{n=1}^{\infty} \mathbf{P}\left(\frac{\ln N_{n}}{m \lambda n}>1+\varepsilon\right)<\infty .
$$

Similarly, we can get that

$$
\sum_{n=1}^{\infty} \mathbf{P}\left(\frac{\ln N_{n}}{m \lambda n}<1-\varepsilon\right)<\infty .
$$

From the above two formulas, we have

$$
\frac{\ln N_{n}}{m \lambda n} \longrightarrow 1, \quad \text { almost surely }
$$

as $n \longrightarrow \infty$. Since $N_{t}$ is nondecreasing in $t$, for any $t \in(n, n+1)$, we have

$$
\frac{\ln N_{n}}{m \lambda n} \cdot \frac{n}{n+1}<\frac{\ln N_{t}}{m \lambda t}<\frac{\ln N_{n+1}}{m \lambda(n+1)} \cdot \frac{n+1}{n} .
$$

From this and (1), the result follows. 


\section{JAVANIAN AND M. Q. VAHIDI-ASL}

\section{REFERENCES}

D. Y. C. Chan, B. D. Hughes, A. S. Leong, W. J. Reed, Stochasticaly evolving networks, Phys. Rev. E, 68 (2003), 24.

B. Chauvin, T. Klein, J. Marckert, A. Rouault, Martingales and Profile of Binary Search Trees. Electronic Journal of Probability, 10 (2005), 420-435.

R. Dobrow, and R. Smythe, Poisson approximations for functionals of random trees. Random Structures Algorithms, 9 (1996), 79-92.

Q. Feng, C. Su, The structure and distances in Yule recursive trees Random Struct. Algorithms, 31 (2007), 273-287.

J. L. Gastwirth, P. K. Bhattacharya, Two probability models of pyramids or chain letter schemes demonstrating that their promotional claims are unreliable. Operations Research, 32 (1984), $527-536$.

H. Mahmoud, R. T. Smythe, A survey of recursive trees, Theory Probab. Math. Statist., 51 (1995), 1-27.

S. M. Ross, Stochastic processes, Wiley, New York, 1983.

Mehri Javanian,

Department of Statistics, Faculty of Sciences, University of Zanjan,

Zanjan, Iran.

e-mail: javanian@znu.ac.ir

Mohammad Q. Vahidi-Asl,

Department of Statistics,

Faculty of Mathematical Sciences,

Shahid Beheshti University, Evin, Tehran,

e-mail: m-vahidi@sbu.ac.ir

Received September 2012 